\title{
Endodontics Generation Next- Microrobotics Category of The Article-Review
}

\author{
Sunandan Mittal ${ }^{1}$ \\ Tarun Kumar ${ }^{2}$
}

Shifali Mittal ${ }^{3}$

Jyotika Sharma ${ }^{4}$

\begin{abstract}
A typical endodontic procedure includes access preparation, root canal preparation and root canal filling. This treatment process is time-consuming and prone to human error. The outcome relies on the clinician's master skills that can be gained only through years of training and practice. In order to improve the quality and reliability of endodontic therapy, an endodontic micro robot is proposed to be built. The objective is to modernize the traditional treatment, moving from a "manual art" to science-based automation. This computer controlled micro machine is mounted on the teeth within patient's mouth. With on-line monitoring and positioning control, the multipurpose micro robot performs automatic treatment procedures, including probing, drilling, filing, cleaning and filling. This article gives an insight to design and applications of the intelligent robotic micro-machine applicable to root canal preparation with online monitoring capabilities that will increase the accuracy and efficiency of the treatment.
\end{abstract}

\section{INTRODUCTION}

There are many problems that can occur in current endodontic techniques for the canal preparation. These include perforations, canal ledging, transportation of the apical portion of the canal and stripping

The success for endodontic therapy is closely related to the technical quality of the root filling [Grahnen and Hansson $^{3}$ 1961, Sjögren et al $\left.{ }^{9} 1990\right]$. Because failures are common, a major portion of a specialist's time is devoted to re-treatment. Unfortunately, re-treatment of failed cases only has about a $65 \%$ success rate even when endodontic specialists supervise or perform re-treatment [Bergenholtz, et al. ${ }^{1}, 1979$, Sjögren, et al. ${ }^{9}$ 1990]. It has been stated that to improve the quality of endodontic treatment in general practice it will be necessary to develop simpler techniques without sacrificing the quality of care.

There is a need for advanced endodontic technology innovation by applying advanced engineering and computer aided technology to reduce the potential for human error and improve the quality of care during endodontic therapy. Advanced endodontic technology development program developed a technique to thoroughly assess the internal tooth anatomy using multiple 2-dimensional $\mathrm{x}$-ray and endoscopic images to build a computer 3-D tooth model, displayed with state-of-the-art computer graphics; developed an automatic prescription system from the 3-D model, using computeraided treatment procedure planning; designed and build a smart multi-purpose precision micro drilling machine that can position and orient itself to start the drilling with intelligent control and on-line monitoring for quality assurance.

\section{ADVANCED ENDODONTIC TECHNOLOGY (fig 1.)}

Advanced endodontic technology was proposed by Dr. Hong ${ }^{5}$. Advanced Endodontic Technology Development project consists of four sub-subjects:

1. Development of a technique to thoroughly assess the teeth's condition using 2-dimensional x-ray images to build a computer 3-D tooth model, displayed with stateof-the-art computer graphics;

2. Develop an automatic prescription system from the 3-D root canal model, using computer aided treatment procedure planning;

3. Design and build a smart multi-purpose precision micro machine to perform automated root canal treatment;

4. Develop a new ultrasonic cleaning tool with pressure assisted jetting/vacuum waste removal.

The development of an endodontic micro machine is the center piece of Advanced Endodontic Technology Development project, as the final implementation of advanced endodontic technology will come down to the micro machine with a precision beyond what the human hand can achieve. This computer-controlled machine will be mounted on the teeth within the patient's mouth. With online monitoring and intelligent control, the micro machine or robot will perform the automated probing, drilling, cleaning, and filling of root canal. All other subproject results are incorporated into this robotic operation.

\section{D COMPUTER MODELING OF ROOT CANALS}

Before a clinician's inspection, a destructive access preparation by removing teeth crown and dentin is usually needed. 3 D Computer Modelling of Root Canals is a nondestructive assessment of the internal anatomy of the teeth.

In this, a less invasive technique and a computer program are developed to assess internal tooth geometry by building 3-D tooth models from 2-D radiographs, within the 
constraints of human body. This model reveals the dimensions and geometry of the root canal, and displays the location of canal orifice and canal curvature in 3-D computer graphics. With this model, the destructive access preparation before a clinician's inspection can be avoided .

\section{COMPUTER-AIDED PRESCRIPTION OF TREATMENT}

Computer-aided prescription of treatment procedures in some sense functions like CAD/CAM programs in the machining industry, in which the tool paths and cutting parameters are generated for $\mathrm{NC}$ (numerical control) programs from computer aided design. The computer-aided process planning system chooses and positions the treatment tools, controls the tool paths and motions for the automated root canal treatment, and output the standard numerical controlled program. An optimization program is integrated into this auto-prescription program to minimize the removal of tooth structure and to meet clinical requirements. This program prevents problems identified with conventional techniques (e.g., inadequate opening, overzealous tooth removal) and identify precise control parameters for the intelligent drilling. The geometry of the 3-D tooth model after treatment can also displayed so that the dentist can review with the patient before actual access preparation and canal drilling starts.

\section{ENDODONTIC MICRO ROBOT}

Specific objectives for micro robot design include: (1) reducing the reliance on the skills of the dentist, (2) minimizing human error, and (3) offering a method for precise diagnosis and treatment.

This machine should have the following features:

A micro-position and orientation adjustment to ensure that the tools start at a precise point;

- An automatic feed rate and travel distance control to ensure that the tools can reach the required canal depth and stop at a designated point;

- Built-in micro sensors to monitor the probing and drilling/reaming process;

- Apex sensing and control to prevent root perforations or the potential to over shoot (Exceeding the apex of the canal);

- Flexible drills or files to allow for cleaning and shaping curved canals;

- Vacuum attachments capable of sucking the debris or loose tissue from the root canal and/or pressurized solution jets to flush the chips away.

- An ideal basic machine must have five degrees of freedom to control the following axes:

- $\quad \mathrm{X}$-axis, along the teeth row, with travel range of $5 \mathrm{~mm}$;

- $\quad$ Y-axis, across the teeth row, with travel range of $4 \mathrm{~mm}$;

- Z-axis, the tool advancement direction, perpendicular to the tooth occlusal surface, with a travel range of $15 \mathrm{~mm}$ minimum. When using a longer tool, the endodontic tool should be able to reach $28 \mathrm{~mm}$ from the tooth crown, covering the required range of treatment;

- The angular adjustment of the tool entrance angle of \pm $12^{\circ}$ in the $\mathrm{X}-\mathrm{Z}$ plane;

- The angular adjustment of the tool entrance angle of \pm $12^{\circ}$ in the $\mathrm{Y}-\mathrm{Z}$ plane.

In addition, the design must meet the following requirements:

- The size of the machine must be compact enough to fit into the patient's mouth and sit on the teeth between his/her two jaws. The dimension should be within 20 $\mathrm{mm} \times 20 \mathrm{~mm} \times 28 \mathrm{~mm}$;

- The spindle must have the rotational power to drive the tool at speeds and torque used in endodontic treatment tools;

- The machine should be able to provide a thrust force not less than $500 \mathrm{~g}$ ( 4.9 Newtons) for tool penetration into the crown and dentin.

Designed for multi-purpose use, this machine can hold various endodontic tools and auxiliary devices. With a quick tool change concept, utilizing a cartridge design, different tools can be pre-mounted on a small modular unit, which can be inserted into a sliding adaptor on the $\mathrm{Z}$ axis. The machine design also incorporates sensors for intelligent monitoring of the treatment process. Because the compact feature of the sensors, it can be fabricated using surface micromachine method into silicon-on-insulator (SOI) wafers and embedded in the micro robot. Six micro actuators will be used to control the five axes (five degrees of freedom) and turn on/off the spindle of the tool. Each actuator is independently controlled by a digital NC controller. The controller should react the sensor input quickly, such as in a few millisecond. Auxiliary functions will be also provided such as: irrigation nozzle for cleaning, vacuum suction cup for chip and waste fluid removal, and/or optical fibers for lighting, imaging and observation. A manual remote control is provided for the clinician, but a fully automatic operation with computeraided treatment procedure planning and control is the ultimate goal for zero-defect operation. An interface system is provided for the clinician to interact with the machine control. 

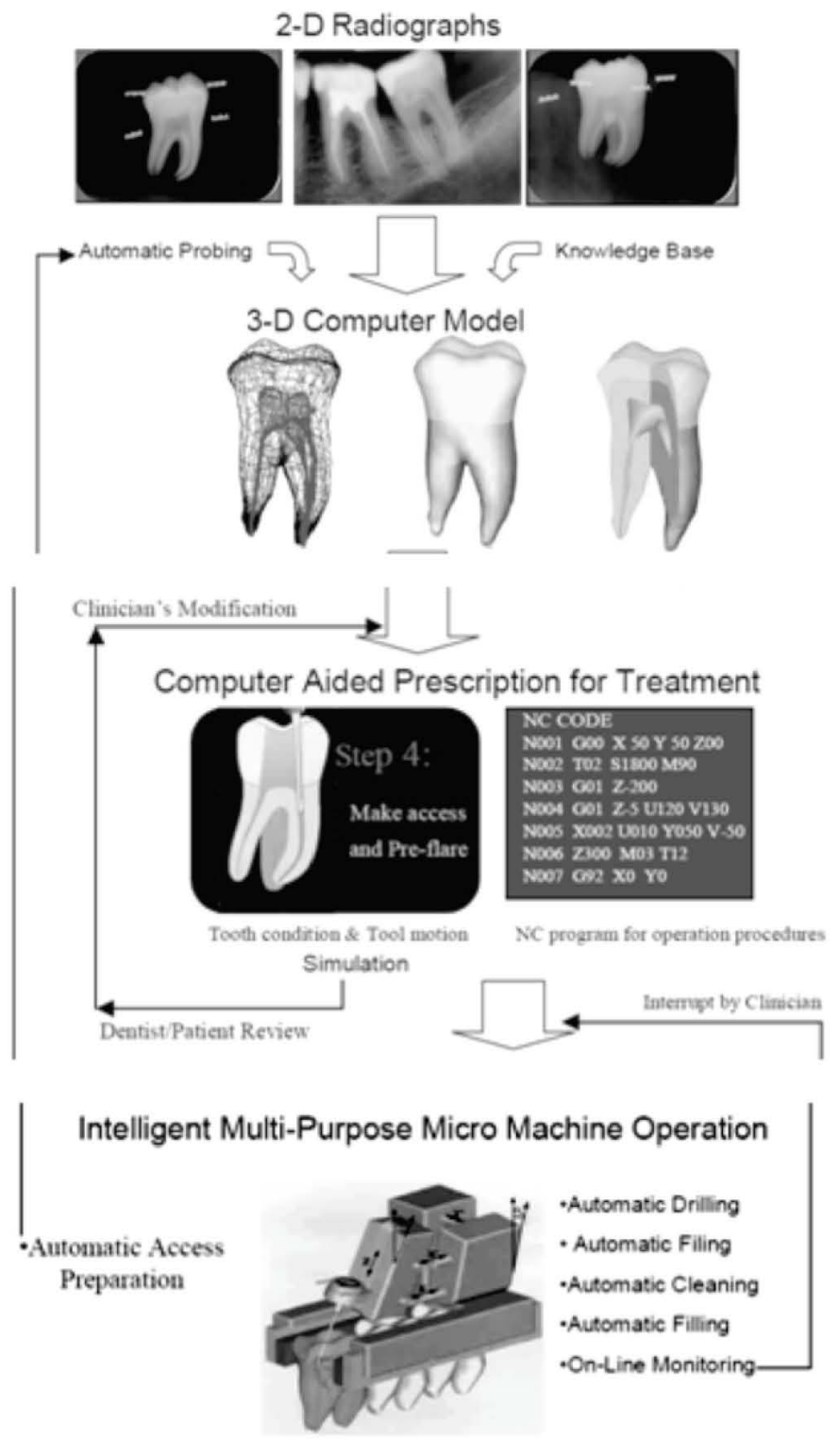

Advanced Endodontic Technology

(Fig.1)

\section{CONCLUSION}

The development of an endodontic micro robot is the center piece of Advanced Endodontic Technology Development.
Compared to conventional access methods for root canal treatment, this technology presents a less invasive method for automated access and canal preparation during endodontic therapy. The automated treatment using micro endodontic robot prevents problems identified with conventional techniques (e.g., inadequate opening, overzealous tooth removal), and provide safe, accurate, and reliable root canal treatment for patients.

According to IADR's press release, saving the dentist from having to lean over could lead to employment opportunities for dentists with spinal disabilities, and the transmission of disease between the dentist and the patient is impossible when the robot is used. Also, the robot could be useful in providing dental care in countries at war or with economic problems.

\section{REFERENCES}

1. Bergenholtz G, Lekholm U, Milthon R, and Engstrom B (1979): Influence of apical over-instrumentation and overfilling on re-treated root canals. J Endodon 5: 310-314.

2. Burns RC and Herbranson EJ: Tooth Morphology and Cavity Preparation. In Cohen S, Burns RC, editors: Pathways of the Pulp, ed 7, St Louis, Missouri, The C. V. Mosby, 1997, pp 150-202

3. Grahnen H ,Hansson L(1961): The prognosis of pulp and root canal therapy .Odontol Revy 121:146-65

4. Gutmann JL, Dumsha T: Cleaning and Shaping the Root Canal System. In Cohen S, Burns RC, editors: Pathways of the Pulp, ed 7 , St Louis, Missouri, The C.V. Mosby Company, 1997, pp160-173

5. Intelligent Micro Robot Development for Minimum Invasive Endodontic Treatment

6. J. Dong, S.Y. Hong, G. Hasselgren (2005): Theories and algorithms for 3-D root canal model construction. Computer-Aided Design 37: 1177-1189.

7. Janet Dong, Shane Hong Gannar Hesselgren WIP: A Study on Development of Endodontic Micro Robot .Proceedings of The 2006 IJME - INTERTECH Conference

8. Shimon Friedman "Treatment outcome: the potential for healing and retained function" in ingle bakeland Ingle Endodontics edition 6th pages 1162-1232.

9. Sjogren U, Hagglund, B, Sundqvist G, and Wing K. (1990): Factors affecting the long-term results of endodontic treatment. J Endodon, 16: 498-504.

10. West JD and Roane JB: Cleaning and Shaping the Root Canal System. In Cohen S, Burns RC, editors: Pathways of the Pulp, ed 7, St Louis, Missouri, The C.V. Mosby, 1997, pp 203-257. 